\title{
The Design and Implementation of Real-Time Bus Monitoring Based on 4G Mobile Internet
}

\author{
Jian $\mathrm{He}^{1, \mathrm{a}}$, Yongtai Zhang ${ }^{1, \mathrm{~b}}$, Mingwo Zhou ${ }^{1, \mathrm{c}}$, Yi Han ${ }^{2, \mathrm{~b}}$ \\ ${ }^{1}$ School of Software Engineering, Beijing University of Technology, Beijing 100124,China \\ ${ }^{2}$ China Welfare Lottery Technology Center, East Street No.73 4th North Ring Road, Chaoyang \\ District, Beijing 100010, China \\ ajianhee@bjut.edu.cn, byt9221@foxmail.com, 'Plegendsister2@163.com
}

Keywords: Bus monitoring; 4G; H.264; RTSP; GPS; FPGA

\begin{abstract}
With the rapid growth of vehicles scale in China, the development of public transportation has become an effective way of controlling urban traffic problems. However, the continuous occurrence of public traffic accidents has caused widespread concern in China. Hence, how to efficiently monitor the security status in the bus is becoming a hot research field. In this paper, the H.264 video compression standard and real-time streaming protocol (RTSP) are introduced to achieve real-time transmission of surveillance video. Besides, a real-time bus monitoring system combining with 4G transmission, GPS, FPGA and network camera technologies is designed to achieve real-time bus video monitoring, positioning, and emergence handling. The system is actually tested in two Beijing bus lines, and is proved by practice that the system can effectively improve the efficiency of bus monitoring and positioning with features such as short video set-up time, clear bus video, and accurate bus positioning.
\end{abstract}

\section{Introduction}

With the rapid development of economy and the fast growth of vehicles scale in China, the traffic control has already become a major problem faced by the urban development process, while an effective method of solving the problem is to greatly develop public transport and improve its service quality. Therefore, the Ministry of Chinese Construction clearly has put forward the "bus priority strategy" to develop public transport in cities. For example, Beijing Bus Company has one of the largest transit systems in China, which operates 1020 routes using a fleet of 22542 buses, and transports more than 4.6 billion passengers in 2013. However, with the rapid increase of buses, the continuous occurrence of public traffic accidents has caused widespread concern in China. As a result, public transport monitoring technology based on modern communication and information technology has already become a hot research field in intelligent transportation systems (ITS) [1]

A variety of sensing modalities has become available for bus monitoring, including radar, GPS, RFID and cameras [2, 3]. Today, the satellite-based GPS is widely used for bus monitoring because the GPS receiver can provide bus position and velocity data in global coordinates. For example, Hu Niu presented the GPS-based Beijing Bus Monitoring System (BJ-BMS) which was equipped with the GPS terminals and GPRS modules, and could tell the location, speed and information of overall distribution of the buses [4]. However, a standalone GPS receiver could not fulfill the station reporting due to the occasional loss of satellite connection and signal errors. To provide continuous, accurate, and high integrity position data, the bus positioning should be aided by additional sensors such as an inertial navigation system (INS), digital road map, RFID, and cameras [5]. Mustapha combined GPS with RFID and GPRS to identify and monitor bus [6]. He Feng proposed a supervisory system based on GPRS and ZigBee technology [7]. Due to the complementary natures of the GPS and INS sensors, the fusion technology of GPS and INS data has been used for bus positioning systems [8].Especially getting along with the digital technology development, the bus video monitoring is transforming from analog into digital storage. Hence, more and more researchers study the bus monitoring technology based on such digital camera. For example, a pair of two-camera omni-imaging devices has properly been designed for use on the top of a video 
surveillance vehicle to monitor passing-by persons [9]. An effective nighttime vehicle detecting and tracking system was proposed for identifying and classifying moving vehicles for traffic surveillance [10]. However, the bandwidth limitation of early mobile communication (such as GSM, GPRS, CDMA and 3G) makes most of the buses install off-line video storage, which could not transmit video data to the bus management center in real time, and could not make a rational bus dispatching. 4G networks have a simple structure based on all-IP where Internet Protocol (IP) packets traverse an access network, and enable IP-based voice, data, and streaming multimedia at higher speeds - theoretically up to $100 \mathrm{Mb} / \mathrm{s}$ for mobile users. Hence, the $4 \mathrm{G}$ is introduced to develop the real-time bus monitoring system in this paper.

The rest of this paper is organized as follows. Section II introduces the H.264 coding standard and Real Time Streaming Protocol (RTSP) at first, and then proposes the architecture of bus monitoring system based on 4G mobile Internet. Section III presents the design and implementation of the system. The system is tested and validated in Section IV, and the conclusion and future research are proposed in Section V.

\section{Foundation}

The stream coding standard and the protocol for stream transmitting play key roles for bus video monitoring, so the H.264 coding standard and RTSP are adopted to implement the bus video monitoring system.

\section{H.264 Coding Standard}

In order to reduce the data of transmission, different compression techniques have been used into video stream coding. Nowadays, main stream coding techniques include MJPEG, MPEG, MPEG-2, MPEG-4 and H.264, etc. In terms of public transport monitoring system, the most concerned factors include clearness, bandwidth and stability .

H.264 video coding standard was proposed by Joint Video Team (JVT) in the 1990s. Compared with previous video coding techniques, H.264 makes a great progress in video compression. In addition, it has lots of advantages, such as high image quality, strong network adaptive capacity, favorable capability of correction, and so on. Hence, it is widely applied in fields of real-time video communication and digital video broadcast.

According to H.264 technical specifications, video compression and network transmission are relatively independent, and they correspond to video coding level (VCL) and network abstract level (NAL) in structural division, respectively. VCL is composed of core compression engine, macro block and level grammar definition. The main objectives of VCL are coding and decoding effectively as much as possible. The main function of NAL is to adapt to bit string produced by VCL, so as to make it adapt to complicated network environment. In addition, NAL also supports for SEI and streaming format code data transfer function [8].

\section{Real Time Streaming Protocol}

Video stream downloading from the server to the client generally experiences several stages of acquiring stream source, coding, transmission, decoding, buffering and playing, etc. In those steps, streaming transmission plays a key role, which requires the support of basic transmission protocol.

RTSP is an application-level protocol for control over the delivery of data with real-time properties. It provides an extensible framework to enable controlled, on-demand delivery of time-synchronized continuous media, and sources of data can include both live data feeds and stored clips. The protocol is intended to control multiple data delivery sessions, provide a means for choosing delivery channels such as UDP, multicast UDP and TCP, and provide a means for choosing delivery mechanisms based upon RTP. The main advantage of RTSP is as follows.

(1) Real time transmission and playing. Media synchronization is performed at the transport level. Each media stream within a presentation can reside on a different server, and the client automatically establishes several concurrent control sessions with the different media servers. RTSP supports frame-level accuracy through the Society of Motion Picture and Television Engineers (SMPTE) time stamps to support the record and playback mode. 
(2) Secure and saving storage space. RTSP re-uses web security mechanisms in order that all HTTP authentication mechanisms are directly applicable. It can be easily parsed by standard HTTP or MIME parsers. Besides, clients have no need to store all download data in the local storage, so as to save lots of storage space [9].

\section{System Architecture based on 4G mobile network}

The real-time bus monitoring system is mainly composed of the Bus Terminal, 4G mobile Internet, the Monitoring Server and the Bus Monitoring Center. Fig.1 shows the system architecture. The data from the monitoring bus can be classified into stream data (such as video data from camera and audio data from microphone), and digital data (such as GPS, temperature, velocity and alarm data). The main module is as follows.

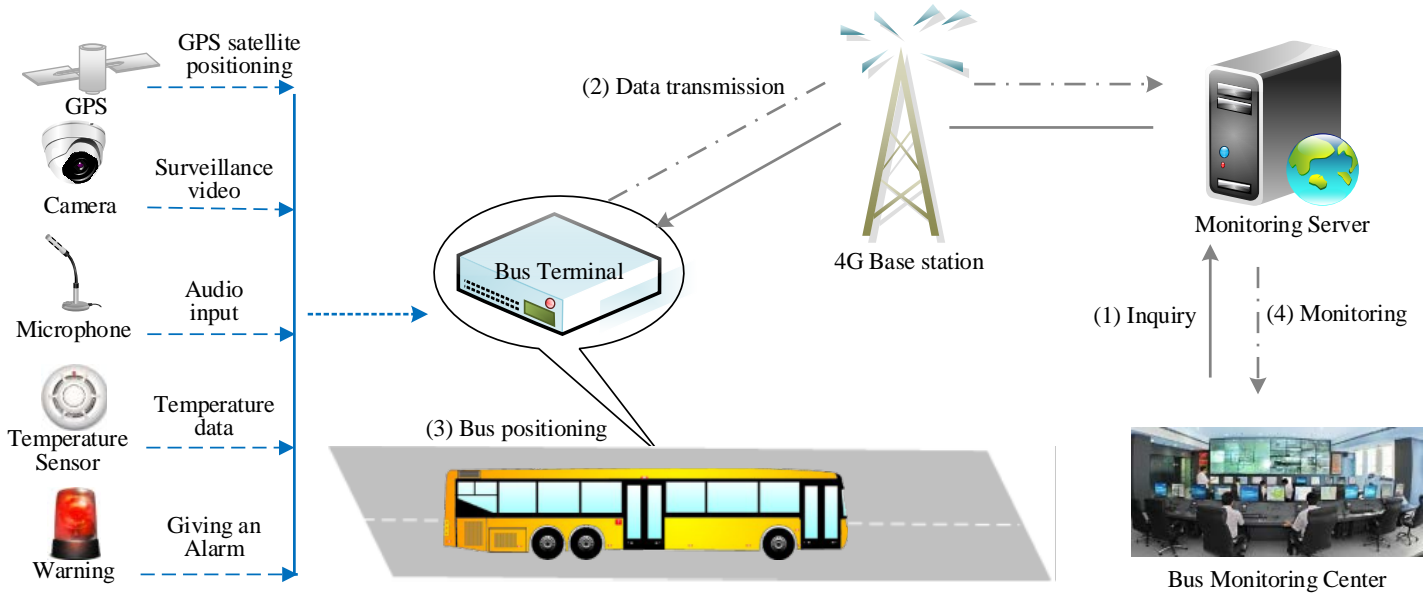

Fig. 1 The Architecture of Bus Monitoring System

Bus Terminal. Bus Terminal which is the onboard unit for positioning and tracking bus. It is mainly consisted of the FPGA-based mainboard (constituted of Zynq 7000 FPGA, the GPS receiver, 4G transceiver module, Flash disk, etc.), display device, and interfaces for connecting different sensors (such as network camera with microphone, temperature and velocity sensors). FPGA-based mainboard is responsible for collecting data and digitalizing compression of video and audio data according to the H.264 standard, and storing the data into flash disk, or transmitting the data to the Monitoring Server. GPS receiver analyses and translates the signals from GPS satellite into GPS data, and then sends the GPS data to the Monitoring Server through 4G transceiver module. Video cameras are installed at important position of the bus (locating at front of the driver, import and export door, and at the tail of the bus), so as to monitor the status of the bus. The display device can receive and show the interactive information. Bus terminal plays a key role for bus monitoring system.

4G Base station. Bus Terminal is consisted of $4 \mathrm{G}$ wireless network, and Internet. 4G wireless network consists of $4 \mathrm{G}$ transceiver module and $4 \mathrm{G}$ base stations. It is responsible for transmitting the collecting data from the Bus Terminal to the Monitoring Server, and receiving commands from the Bus Monitoring Center. Besides, it is also responsible for downloading video/audio stream from the Bus Terminal to the Bus Monitoring Center.

Monitoring Server. Monitoring Server which is the hub of the bus monitoring system. It receives digital data (such as GPS, velocity, temperature etc.), and responds the retrieving requirement from the Bus Monitoring Center so as to show the real-time state about all of the running bus. Besides, it forwards the play requirement from the Bus Monitoring Center to the network camera connecting with the Bus Terminal, in order to play the video of the bus.

Bus Monitoring Center. Bus monitoring center which receives the input options from user, and real-time displays the status about all of the running buses. For example, it can show the position of the bus, and report its previous and next stations according to the GPS and GIS data. It can also real-time display the video stream received from the monitored buses at the same time.

The process which locates a bus and displays its video stream can be divided into four steps, namely inquiry, transmission, bus positioning, and monitoring.

(1) Inquiry:The user issues positioning and video monitoring requests by selecting a bus and its 
camera ID from the Bus Monitoring Center. And the requirement is sent to the Monitoring Server.

(2) Transmission:According to the number of the selected bus and its camera ID, the Monitoring Server forwards the request command to the specific Bus Terminal through 4G mobile network.

(3) Bus positioning:The Bus Terminal collects the digital data from connecting sensors and sends them back to the Monitoring Server as soon as it gets the request.

(4) Monitoring:The Bus Monitoring Center shows the running status of the selected bus, and plays its video stream. For example, it shows the position, the temperature and velocity of the selected bus, and real-time plays its video stream.

\section{Design and Implementation}

Based on the above architecture, we design and implement the Bus Terminal, the Monitoring Server and the Bus Monitoring Center.

Design and Implementation of Bus Terminal

The Zynq-7000 FPGA from Xilinx is introduced to develop the Bus Terminal, which is mainly composed of FPGA mainboard, data collection module, FPGA processing core and interface. Fig. 2 shows the hardware structure of the Bus Terminal. The main function of each part is as follows.

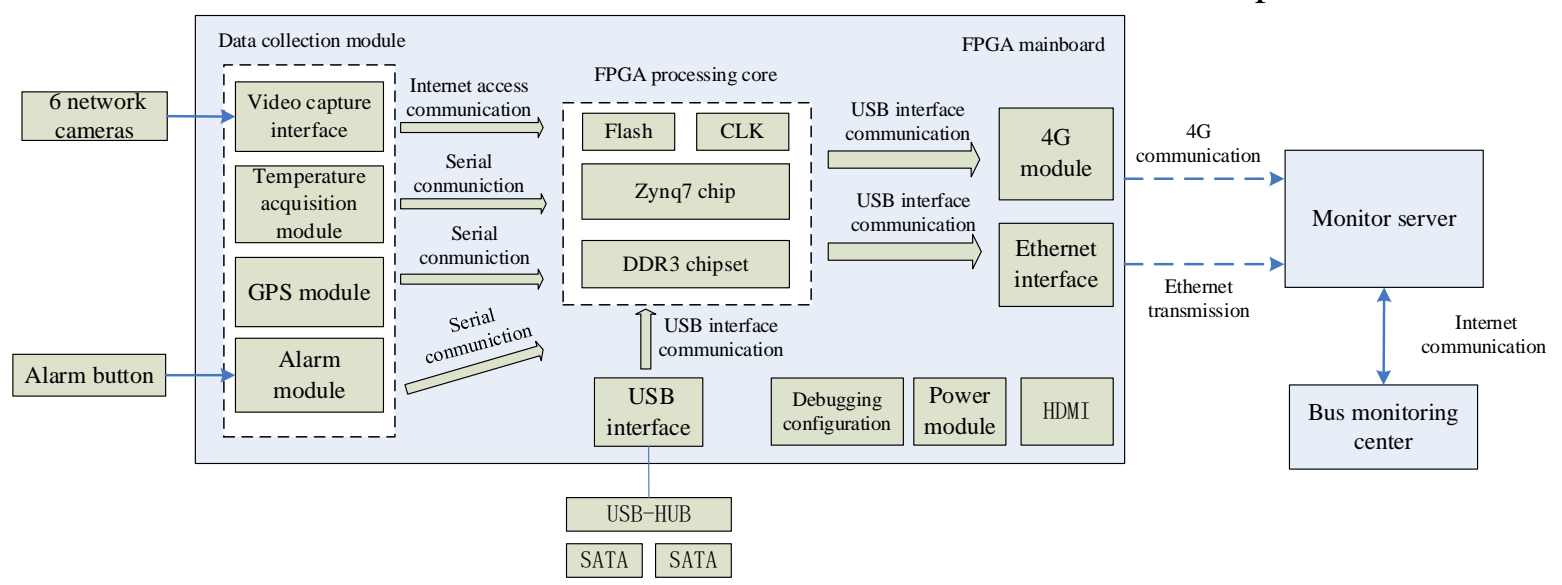

Fig. 2 The hardware structure of the Bus Terminal

(1) FPGA mainboard, which includes several kinds of buses, such as EISA, I2C, and Universal Serial Bus, etc. The buses provide different interfaces to collect different sensors and devices. For example, there are 6 Ethernet interfaces for connecting 6 network cameras, a USB interface for connecting with 4G transceiver module, and an I2C interface for connecting the alarm button. With the FPGA mainboard, the FPGA processing core can communicate with the data collection module and other devices. Besides, there is a power module integrated on the FPGA mainboard, it transfers the power from DC $24 \mathrm{~V}$ provided by the bus into $9 \mathrm{~V}$.

(2) Data collection module which mainly includes 6 network cameras, a temperature sensor, a GPS receiver, and an alarm button. DS18b20 temperature sensor is integrated to collect environmental temperature in the bus, and NEO-6M GPS receiver from Ublox is applied to collect the position data. An alarm button connecting with the FPGA mainboard will issue alert as soon as the bus driver pushes it in times of emergency.

(3) FPGA processing core Zynq XC7Z020 which mainly includes a Cortex A9 processor, 16MB QSPI Flash, and 512MB DDR3 is adopted to develop FPGA processing core. The FPGA processing core which takes charge of data processing and transmission plays the key role of the Bus Terminal. It can receive video data from 6 network cameras through the Ethernet interfaces, and receive temperature data through GPIO interface or position data through GPS receiver. Besides, the FPGA processing core can transmit the data collected from the collection module to the Monitoring Server through 4G transceiver module.

Design and Implementation of the Monitoring Server

According to above functions, this server is mainly composed of control server, data server and video server. Control server is responsible for the authentication both from Bus Terminal and Bus Monitoring Center, maintaining network connection and data transmission control. Data server is responsible for receiving and storing collection data from the Bus Terminal. Video server takes 
charge of receiving video streaming from the Bus Terminal, and forwarding the video stream to the Bus Monitoring Center.

Being the hub of the bus monitoring system, the Monitoring Server must communicate with the Bus Terminal and the Bus Monitoring Center. There are three kinds of exchange data, of which are control command, digital data and video stream. So three ports are designed to support those exchange data respectively (namely control port, data port and video port).

Tab 1 Data packet structure

\begin{tabular}{cccc}
\hline Fields & Types & Length & Description \\
\hline Initial ID & CHAR & 1 & ASCII character “[” \\
Information type & BYTE & 1 & type of the information \\
Information ID & N_STRING & 5 & Information identifier \\
Information content & STRING & variable & information content depending on the protocol specification \\
End ID & CHAR & 1 & character “]” \\
\hline
\end{tabular}

Being a lightweight data-interchange language, JSON is easy for machines to parse and generate. So it is adopted as the data-interchange language among the Bus Terminal, the Monitoring Server and the Bus Monitoring Center. Each data package based on JSON at least includes initial identifier, information type (such as CMD for GPS information, SWD for temperature information, and CLR for alarm information), information ID, information content and end identifier. Table 1 shows the structure of the data package.Take a temperature data package for example. The initial temperature information based on JSON is shown as follows.

[00002(CWD, 02585720.5 091114)]

After parsing the package, the information obtained is as follows.

Information type: Temperature (CWD)

Temperature date: November 09th, 2014(Greenwich Mean Time)

Temperature time: 02:58:57(Greenwich Mean Time)

Temperature: 20.5

Design and Implementation of the Bus Monitoring Center

The Bus Monitoring Center mainly includes GPS positioning module and real-time video monitoring module. The GPS positioning module is mainly responsible for sending request package to the Monitoring Server, and then receiving the response, and displaying the bus status based on GIS. Fig. 3 shows the flow chart of the GPS positioning module.

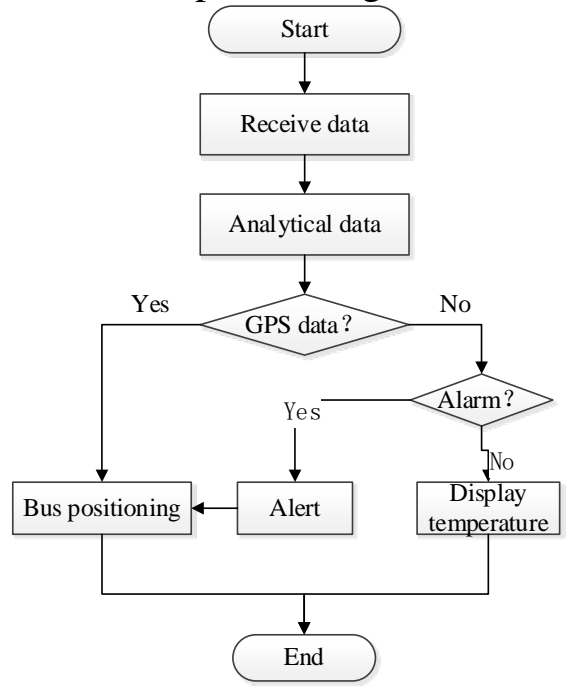

Fig. 3 The flow chart of GPS positioning module

The main function of the GPS positioning module is as follows.

(1) Bus positioning and trace. It acquires the GPS data of the bus stored in the Monitoring Server, and displays the bus position according to the GPS data in Baidu map. It helps user to position and trace the route of a bus.

(2) Inquiry bus status. By inputting the license of a bus, user can easily inquire the real-time status information of the bus. Such as the position, alarm and temperature, etc. 
(3) Inquiry the historical track of a bus. By inputting the license, the start and end time about a bus, the historical track of the bus among the specific time can be marked on the Baidu map.

(4) Multi-bus monitoring. All of the running buses will be shown in the Baidu map according to its GPS data, and the position of each bus could be updated in real time.

The real-time video monitoring module is responsible for sending request package to the Monitoring Server according to the number of the selected bus and its camera ID, and playing the video stream of the selected bus in the screen. User can monitor the real-time status of the bus by playing the bus monitoring video. Since H.264 and RTSP are adopted to code and transmit the video stream, the nVLC which is .NET packaging version of VLC and supports for all kinds of streaming protocols, is introduced to develop the real-time video monitoring module. Fig. 4 shows the flow chart which the Bus Monitoring interacts with the Monitoring Server and Bus Terminal to play the bus video stream.

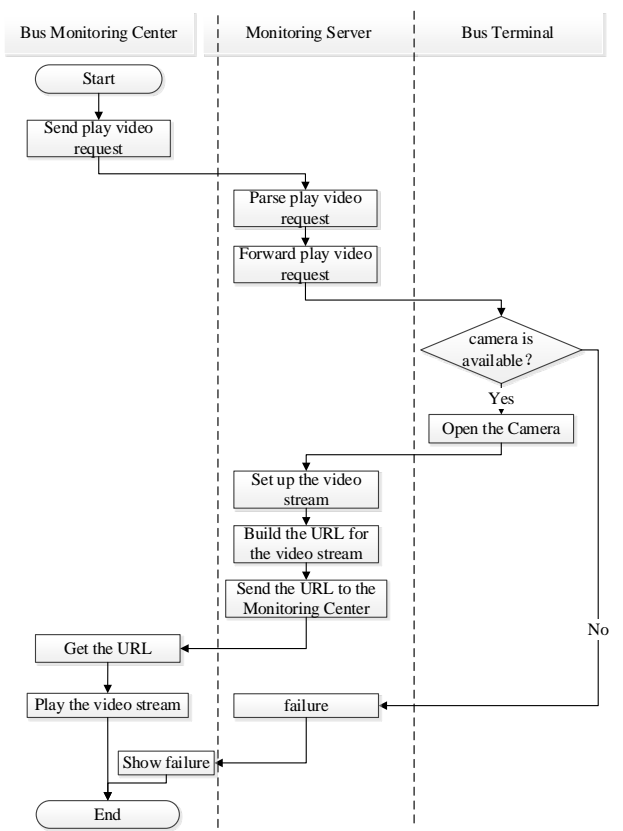

Fig. 4 Flow chart of the real-time video monitoring module

\section{Experiment}

The bus monitoring system is running on BRT03 and No. 607 bus lines belonging to Beijing Bus Company. There are totally 40 buses which have installed the Bus Terminal. The Monitoring Server running on Lenovo x3650M5-D2C is placed in the Data Center at Yizhuang. The software of the Monitoring Center is installed on workstations at Andingmeng bus dispatching center and the Embedded Software and Systems Institute in Beijing University of Technology, respectively.

Video transmitting test

Tab 2. Video transmitting test results under Unicom 4G and 3G

\begin{tabular}{ccccc}
\hline Network type & $\begin{array}{c}\text { Video average } \\
\text { transmission rate(kb/s) }\end{array}$ & $\begin{array}{c}\text { Video Average } \\
\text { set-up time }(\mathrm{s})\end{array}$ & $\begin{array}{c}\text { Video code } \\
\text { rate }(\mathrm{kbps})\end{array}$ & $\begin{array}{c}\text { Average lagging } \\
\text { times(times/min) }\end{array}$ \\
\hline 4G & $1790 \sim 2096$ & 9.27 & $408 \sim 480$ & 5.3 \\
$3 \mathrm{G}$ & $412 \sim 536$ & 12.21 & $101 \sim 144$ & 13.9 \\
\hline
\end{tabular}




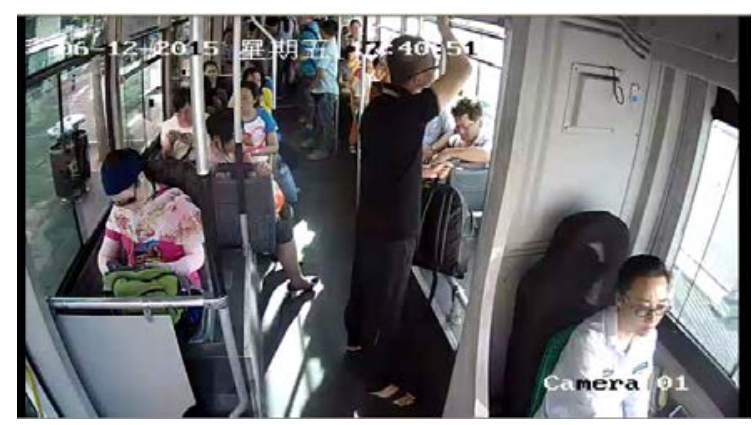

(a) Video Image on Unicom 4G

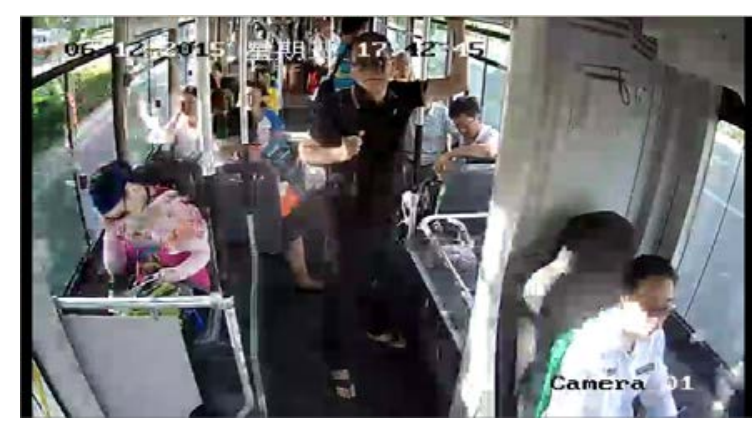

(b) Video Image on Unicom 3G

Fig. 5 Video quality comparison between Unicom 4G and 3G

As shown in table 2, the video average set-up time which starts from the Monitoring Center issuing play video requirement, and ends its receiving the video stream from the Bus Terminal is less than 10 seconds. Besides, the video average transmission rate and video code rate on 4G are almost 4 times better than those on 3G, and the average lagging times on 4G is 2.62 times less than those on 3G. Fig. 5 indicates that the video quality on 4G is better than those on 3G. Besides, the video average establishment time on $4 \mathrm{G}$ is less than those on 3G. The experiment indicates that the bus monitoring system based on $4 \mathrm{G}$ can ensure video transmission quality with clear and fluent video frame.

Positioning test

Since the Bus Terminal is integrated with GPS receiver, it makes the system can position all of the running buses in a specific bus route in real time. Besides, combining with GIS, all of the running bust can be shown in the specific route at the same time, and it can help bus dispatching center to judge the crowded status according to the number of running buses between two bus stations. Taking route 607 for example, there are about 20 buses at the peak time from 8:00 to 9:00 am on June 13 2015. Fig. 6 shows the crowded status at a part of the route 607, of which is from Anzhenxili station to Heping East Bridge station. The crowded status is divided into three grades, namely red, orange and green from the high-low in order. The red one means that there are at least 3 buses in the section, orange represents that there are about 2 buses in the section, and green indicates that there is less than 2 buses in the section. Fig. 7 extracted from Baidu map also shows the traffic status from Anzhenxili station to Heping Bridge east station at the same time, in which the red section means very crowded, orange section stands for crowded traffic, and green section indicates smooth traffic. It is obvious that the crowded status shown in Fig.7 is quiet similar to the status shown in Fig.6.

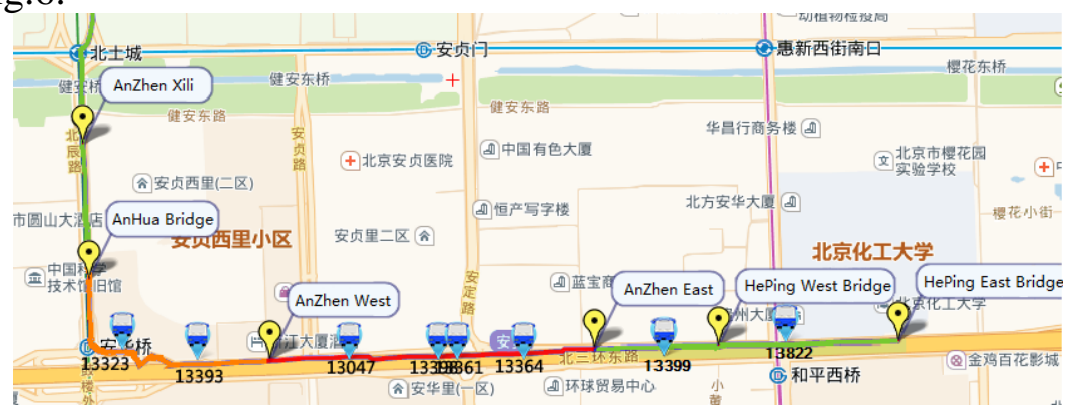

Fig. 6 The crowded status from Anhua North to Heping East Bridge station

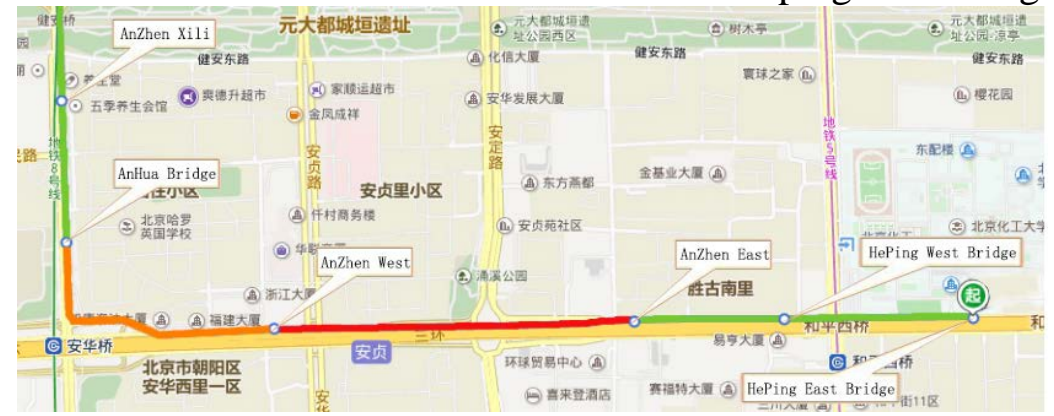

Fig. 7 Baidu map shows the crowded status from Anhua North to Heping East Bridge station 


\section{Conclusions}

This paper studies the safety monitoring technology of public transport based on mobile internet, and proposes a bus monitoring system architecture based on 4G mobile Internet. In order to realize the real-time positioning and video monitoring bus, the FPGA, GPS, network camera and 4G technologies are introduced to develop the Bus Terminal. Besides, the H.264 coding standard and RTSP are adopted to build a real-time bus monitoring system. The system is actually tested in two Beijing bus lines, and is proved by practice that the system can effectively improve the efficiency of bus monitoring and positioning with features such as short video set-up time, clear bus video, and accurate bus positioning.

In the future, we will further enrich and improve systematic functions. Such as improving video automation identification and network adaptive ability, and developing decision support system for bus dispatching, etc.

\section{Acknowledgment}

This paper is supported by project of Natural Science Foundation of China (No. 61040039, and partly supported by project of Beijing Natural Science Funds (No. 4102005).

\section{Reference}

[1] M. Cheon, W. Lee, C. Yoon, M. Park. Vision-based vehicle detection system with consideration of the detecting location. IEEE Trans. Intell. Transp. Syst., vol. 13, no. 3, pp. 1243-1252, Sep. 2012.

[2] C. Oberli, M. Torres-Torriti, D. Landau. Performance Evaluation of UHF RFID Technologies for Real-Time Passenger Recognition in Intelligent Public Transportation Systems. IEEE Trans. Intell. Transp. Syst., vol. 11, no. 3, pp. 748-753, Sep. 2010.

[3] J. Kichun, C. Keounyup, S. Myoungho Sunwoo. Interacting Multiple Model Filter-Based Sensor Fusion of GPS With In-Vehicle Sensors for Real-Time Vehicle Positioning. Intell. Transp. Syst., vol. 13, no. 1, pp.329-343, March, 2012.

[4] Hu Niu, Wei Guan, Jihui Ma. Design and Implementation of Bus Monitoring System Based on GPS for Beijing Olympics [C]. 2009 WRI World Congress on Computer Science and Information Engineering, pp. 540-544, July, 2009.

[5] V. J. Hodge, S. O’Keefe, M. Weeks, A. Moulds. Wireless Sensor Networks for Condition Monitoring in the Railway Industry: A Survey. IEEE Trans. Intell. Transp. Syst., vol. 16, no.3, pp. 1-19, March, 2015.

[6] A. M. Mustapha1, M. Hanna2, A. Hussain. Implementing GIS in Bus Identification and Monitoring System. International Conference on Electrical, Control and Computer Engineering, pp. 460-465, June, 2011.

[7] He Feng, Li Lulu, Yin Heng, Huang Xia. Bus Monitoring System Based On ZigBee And GPRS. 2012 International Conference on Computer Distributed Control and Intelligent Enviromental Monitoring, pp. 178-181, Dec. 2012.

[8] R. Toledo-Moreo, M. A. Zamora-Izquierdo. IMM-based lane-change prediction in highways with low-cost GPS/INS. IEEE Trans. Intell. Transp. Syst., vol. 10, no. 1, pp. 180-185, Mar. 2009.

[9] Y. Pei-Hsuan Yuan, Y. Kuo-Feng Yang, T. Wen-Hsiang. Real-Time Security Monitoring Around a Video Surveillance Vehicle With a Pair of Two-Camera Omni-Imaging Devices. IEEE TRANSACTIONS ON VEHICULAR TECHNOLOGY, VOL. 60, NO. 8, pp.3603-3614, OCT. 2011.

[10]C. Yen-Lin, W. Bing-Fei, H. Hao-Yu Huang, F. Chung-Jui. A Real-Time Vision System for Nighttime Vehicle Detection and Traffic Surveillance. IEEE TRANSACTIONS ON INDUSTRIAL ELECTRONICS, VOL. 58, NO.5, pp. 2030-2044 MAY 2011. 